\title{
Alineación frontal de la rodilla. Concepto y determinación de la posición neutra en el vivo
}

Drs. CASAL MORO, R.; MONTERO FURELOS, L.; GONZÁLEZ GARCÍA, J. y PICHEL MOURE, C.M. Servicio de Traumatologia y Cirugia Ortopédica. Hospital Do Meixoeiro. Vigo

\section{RESUMEN}

Se estudiaron 28 rodillas asintomáticas con objeto de confirmar en el vivo la existencia de movilidad mediolateral, tomando como posición neutra aquella en la que el contacto articular se produce simultáneamente en ambos compartimentos. Hemos observado, que la interlínea articular en posición neutra es sensiblemente mas amplia en el compartimento lateral, en proporción de 1,3 a 1 respecto del medial.

Se estudió la rodilla en diversas actitudes posturales durante la estación bípeda, hallando que al colocar la extremidad inferior en adducción de la cadera la altura de la interlínea aumenta significativamente en el compartimento lateral, alcanzando una proporción de 1,9 a 1 respecto del medial. Es decir, que en distintas actitudes fisiológicas, la amplitud de la interlínea articular de la rodilla y por tanto el ángulo fémorotibial, son variables, lo cual es compatible con la hipótesis del «Contacto articular alternativo».

Creemos que la existencia de cierta movilidad mediolateral fisiológica en la rodilla, puede tener trascendencia en el diseño de la geometría frontal de las prótesis, así como en la realización de las técnicas radiográficas y su valoración.

Palabras clave: biomecánica, rodilla, cinemática.

\section{SUMMARY}

There were studied 28 asintomathic knees with object to confirm in the living the existance of medium-lateral movility, taking as a neutral position that in which the joint contact is produced at the same time in both comparments. We have observed, that the join interline in neutral position is slightly wider in the lateral comparment, in a proportion of 1,3 to 1 respect to the medium.

The knee in differnt positions was studied during the biped station, finding that when putting the lower extremity in adduction of the waist the interline hight increases significativelly in the lateral comparment, reaching a proportion of 1,9 to 1 in relation to the medium. That means, that in different phisiological attitudes, the width of the joint interline of the knee and therefore of the femoral-shin-bone angle, are variable, which is compatible with the hypothesis of the "Alternative joint contact".
We believe that the existance of a certain phisiological medium-lateral movility in the knee, can have a certain trascendence in the design of the front geometry of the prosthesis, and the realization of the radiographical techniques and its valoration.

Key words: Biomechanic, knee, kinematics.

\section{INTRODUCCIÓN}

Se admite generalmente que la rodilla posee un movimiento fundamental de flexoextensión, que se realiza en el plano sagital, y movimientos de rotación, de menor amplitud, en el plano horizontal. Clásicamente no se describen o se presta escasa atención a la movilidad mediolateral de la rodilla en condiciones fisiológicas; se presupone también, que ambos cóndilos mantienen un contacto permanente con ambos platillos tibiales durante la actividad habitual del individuo. $1,2,3$

Estudios realizados en rodillas cadavéricas ( $\mathrm{Pichel}^{5}$ ) sugieren que existe un rango fisiológico de movilidad mediolateral que implicaría la alternancia de contacto en uno y otro compartimentos. Este hecho tendría repercusión no sólo sobre el conocimiento básico de la físiología de la rodilla sino también en relación al diseño y técnica de colocación de las prótesis, por lo que pensamos que su confirmación en el vivo tiene gran interés. Por este motivo, se diseñó el presente trabajo, cuyo objetivo fué estudiar las variaciones de la amplitud de la interlínea articular radiológica en rodillas asintomáticas de sujetos vivos, manteniendo distintas actitudes posturales durante la estación bípeda, y tomando como referencia la posición neutra, cuyo concepto definimos como aquella posición de la rodilla en la que el contacto articular se produce simultáneamente en ambos compartimentos.

\section{MATERIAL Y MÉTODOS}

Hemos analizado 28 radiografías de rodilla de individuos asintomáticos de ambos sexos y edades comprendidas entre los 19 y 40 años. Las radiografías se realizaron, adoptando posiciones estáticas normales en la actividad diaria, y manteniendo la articulación en extensión para garantizar unas condiciones uniformes en toda la serie. 
TABLA 1

\section{SERIES RADIOGRAFICAS REALIZADAS}

Serie A: Rodilla en posición neutra 14 casos

\begin{tabular}{ll}
\hline Serie B: Posición de abducción & 7 casos \\
\hline Serie C: Posición de adducción & 7 casos \\
\hline
\end{tabular}

Se estudió la rodilla en tres posiciones estáticas diferentes, neutra, abducción y adducción del miembro inferior, obteniendo tres series de radiografías (tabla 1).

\section{REALIZACIÓN DE LAS RADIOGRAFÍAS}

En todos los casos, se realizó una proyección anteroposterior con $5^{\circ}$ de inclinación craneocaudal, con objeto de incidir más tangencialmente a los platillos tibiales.

- Las radiografías en posición neutra, se obtuvieron colocando al sujeto en decúbito supino e indicándole que contrajera el cuádriceps. El efecto centralizador de este músculo, cuya línea de tracción pasa entre ambos compartimentos, y la ausencia de fuerzas abductoras o adductoras durante el decúbito supino, producirá el contacto articular simultáneo en ambos compartimentos (fig. 1).

- Las radiografías en abducción, se realizaron en bipedestación manteniendo las extremidades inferiores separadas unos $45^{\circ}$, de forma que la reacción de apoyo al peso corporal en cada extremidad, produce sobre la rodilla un momento abductor o valguizante (fig. $2 b$ ).

- Por último, para las radiografías en abducción se colocó al sujeto en bipedestación asimétrica, desplazando la carga corporal hacia la extremidad del lado estudiado, colocando la cadera de ese lado en adducción, de forma que la reacción de apoyo al peso corporal produce sobre la rodilla un momento adductor o varizante (fig. 2c).

\section{VALORACIÓN DE LAS RADIOGRAFÍAS}

Para valorar las radiografías con mayor exactitud, las hemos ampliado proyectándolas (mediante un proyector de transparencias) sobre grandes láminas de papel en las que dibujamos el contorno articular.

Todas las radiografías fueron analizadas, tomando los siguientes puntos de referencia: se trazó una línea LMf tangente al borde distal de los cóndilos femorales y desde los puntosde tangencia lf y Mf de ambos cóndilos, se trazaron sendas perpendiculares hacia los platillos tibiales; se determinaron los puntos de intersección con el contorno del platillo tibial correspondiente, Mt y Lt; en los casos en que el platillo tibial presentaba un doble contorno, se tomó el punto equidistante entre ambos. Se trazó la línea LMt, definida por los puntos Mt y Lt (fig. 3).

Se midió la amplitud de la interlínea en cada compartimento determinando las distancias 1 y m entre los puntos condíleos Lf y Mf y los respectivos puntos tibiales

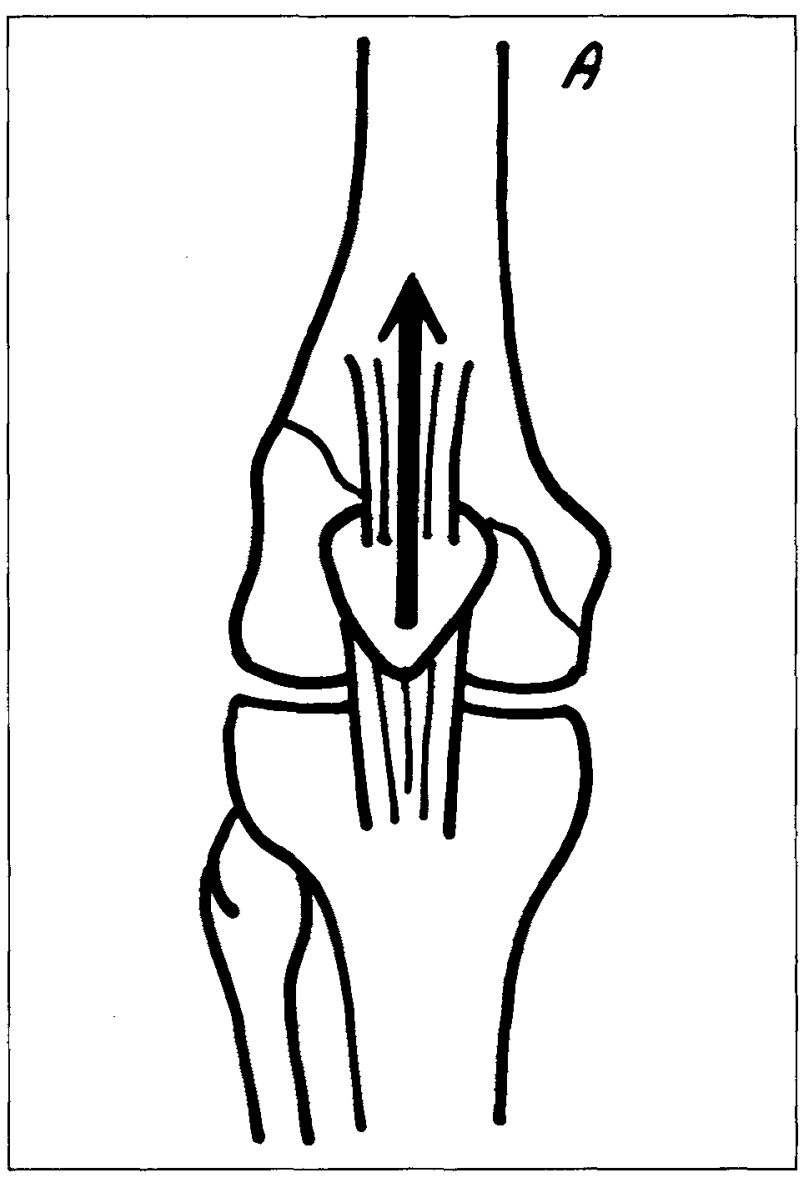

Fig.1. La acción del cuádriceps -cuya línea de tracción pasa en el plano frotal entre ambos compartimentos- garantiza, en decúbito, el contacto bicompartimental.

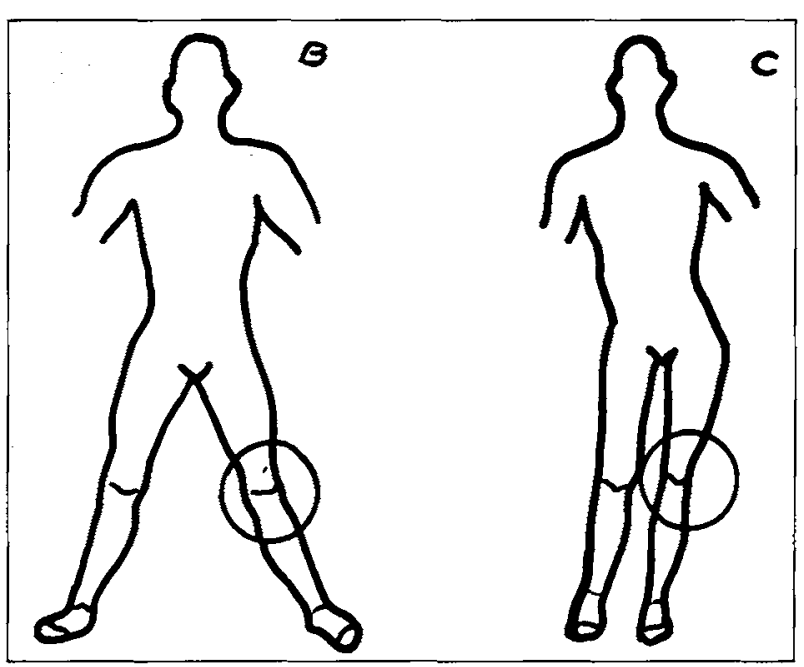

Fig. 2. Colocación del sujeto para realizar las radiografias en las posiciones de abducción y adducción. 
TABLA 2

VALORES DEL COEFICIENTE DE CONVERGENCIA ARTICULAR. $\mathrm{m} /$

\begin{tabular}{l|c|c|c|c}
\hline $\begin{array}{l}\text { Series } \\
\text { radiográficas }\end{array}$ & \multicolumn{1}{c}{$\begin{array}{c}\text { Número de } \\
\text { casos } n\end{array}$} & \multicolumn{1}{c}{$\begin{array}{c}\text { Media } \\
\text { aritmética }\end{array}$} & \multicolumn{1}{c}{$\begin{array}{c}\text { Resviación } \\
\text { standard }\end{array}$} \\
\hline A: Posición neutra & 14 & 1,36 & $0,86-1,92$ & 0,31 \\
\hline B: Posición de abducción & 7 & 1,29 & $0,92-1,74$ & 0,23 \\
\hline C: Posición de adducción & 7 & 1,95 & $1,47-2,61$ & 0,36 \\
\hline
\end{tabular}

Mt y Lt. Para evitar errores a consecuencia de posibles diferencias de magnificación, hemos transformado las medidas realizadas en un coeficiente que relaciona la amplitud de la interlínea en uno y otro compartimento al que hemos denominado Coeficiente de convergencia articular (cociente $1 / \mathrm{m})$; este coeficiente, tendrá como valor la unidad cuando exista un perfecto paralelismo articular, será superior a 1 cuando la interlínea presente una apertura hacia el lado lateral, y menor de 1 cuando éste abierta hacia el lado medial.

Para valorar la apertura de la interlínea articular hemos medido el ángulo $B$, o ángulo de convergencia articular, formado entre la línea LMf y la línea LMt.

- Al tratarse de líneas casi paralelas, la medición directa del ángulo formado ofrece poca exactitud, por lo que hemos recurrido a calcularlo mediante funciones trigométricas a partir de la medición de la distancia entre los puntos Lf y Mf y la diferencia entre los valores 1 y m (altura de los compartimentos lateral y medial) (fig. 3).
Los valores del coeficiente $1 / \mathrm{m}$ (tabla 2 ) y del ángulo $\beta$ (tabla 3), se analizaron estadísticamente en cada una de las series radiográficas, y se establecieron comparaciones entre las tres series para determinar si fistían diferencias estadísticamente significativas.

\section{RESULTADOS}

Hemos encontrado para el Coeficiente de convergencia articular $\mathrm{m} / 1$, los valores señalados en la tabla 2 , donde podemos ver que la media aritmética de dicho coeficiente en posición neutra ha sido 1,36 , con desviación estandard de 0,31 .

Hemos hallado diferencias estadísticamente significativas en el valor de este coeficiente entre la serie $C$ (posición en adducción) y las otras dos series, $(\mathrm{p}<0,01)$ aplicando el test de Student para comparación de pequeñas muestras.

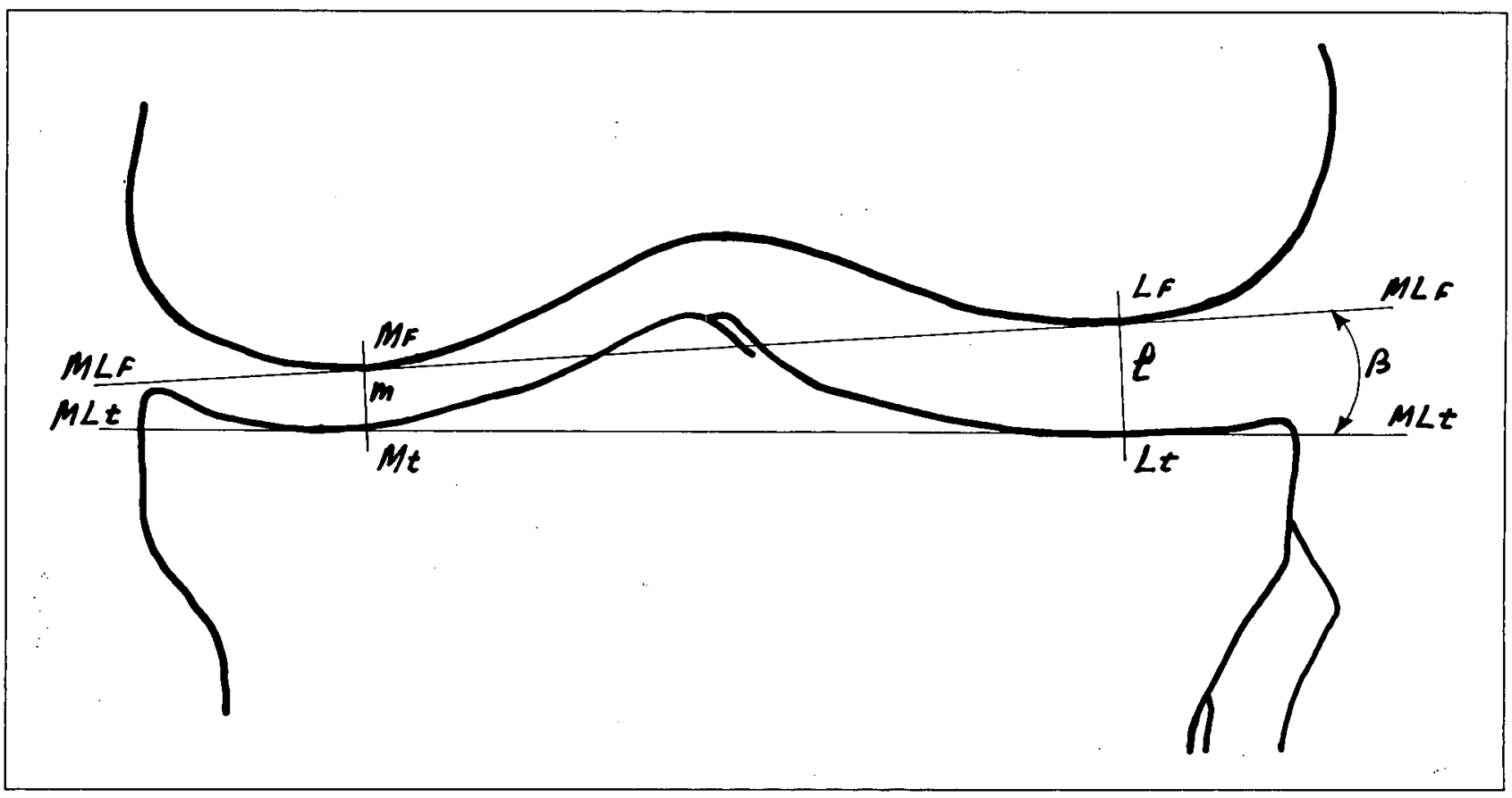

Fig. 3. Método utilizado para medir las radiografias. Explicación en el texto. 
TABLA 3

VALORES DEL COEFICIENTE DE CONVERGENCIA ARTICULAR. (angulo B)

\begin{tabular}{l|c|c|c|c}
\hline $\begin{array}{l}\text { Series } \\
\text { radiográficas }\end{array}$ & \multicolumn{1}{c}{$\begin{array}{c}\text { Número de } \\
\text { casos } n\end{array}$} & \multicolumn{1}{c}{$\begin{array}{c}\text { Media } \\
\text { aritmética }\end{array}$} & \multicolumn{1}{c}{$\begin{array}{c}\text { Resviación } \\
\text { standard }\end{array}$} \\
\hline A: Posición neutra & 14 & 1,1 & $-0,5-2,8$ & 0,99 \\
\hline B: Posición de abducción & 7 & 0,9 & $-0,3-2,4$ & 0,77 \\
\hline C: Posición de adducción & 7 & 2,4 & $1,3-3,3$ & 0,63 \\
\hline
\end{tabular}

Respecto a la apertura de la interlínea, para el ángulo de convergencia articular -ángulo $ß$ - hemos encontrado los valores medios que se expresan en la tabla 3 . El valor angular de la interlínea en la posición de adducción, difiere con significación estadística del encontrado en la posiciones neutra y de abducción ( $\mathrm{p}<0,01$ y $\mathrm{p}<$ 0,02 respectivamente)

\section{DISCUSIÓN}

Con el sistema de medición utilizado, en posición neutra o de apoyo bicompartimental, la interlínea articular es ligeramente más maplia (1,3 veces) en el compartimento lateral que en el medial (véase tabla 2), hallazgo ya constatado por otros autores ${ }^{4}$. Esta diferencia sugiere un mayor grosor del conjunto menisco-cartílagos en el lado lateral. En consecuencia, en la posición neutra, la interlínea articular no es absolutamente paralela, sino que presenta una apertura de $1,1^{\circ}$ hacia el lado lateral.

En extensión completa, y en actitudes de bipedestación, todas ellas fisiológicas, las fuerzas varizantes que actúan sobre la rodilla al colocar la extremidad en adducción, abren sensiblemente la interlínea por el compartimento lateral, cuya altura respecto al lado medial pasa de un valor de 1,3 para la posición neutra a 1,9 para la posición de adducción, en las observaciones realizadas por nosotros, o lo que es lo mismo: de 1,1 a 2,4 grados de apertura lateral.

Sin embargo con el tamaño de la muestra estudiada, no hemos podido encontrar variaciones significativas en la amplitud de la interlínea entre la posición de abducción y la neutra.

En la experiencia que presentamos, creemos demostrar la existencia de movilidad mediolateral para las fuerzas variantes en situación de extensión completa.

Creemos, que en distintas actitudes fisiológicas, la amplitud de la interlínea y consecuentemente, el ángulo fémorotibial son variables. Tales variaciones, que pueden encontrarse minimizadas por el mantenimiento de la rodilla en extensión y el efecto centralizador de la contracción muscular, difícil de evitar cuando se instruye al sujeto para adoptar la posición requerida, son compatibloes con la hipótesis del «Contacto articular alternativo». 5

La existencia de estos movimientos de la rodilla en condiciones fisiológicas nos obliga a conceder mayor importancia a ciertos aspectos del diseño de la geometría frontal de las prótesis de rodilla. También creemos que estos hallazgos pueden tener trascendencia en relación con la realización de las técnicas radiográficas y su posterior valoración, ya que distintos grados de separación de los miembros inferiores y diferentes estados de contracción muscular pueden producir variaciones aleatorias en el ángulo fémorotibial radiográfico.

\section{BIBLIOGRAFÍA}

1. Kapandji, I.A.: Cuadernos de fisiología articular; Toray-Masson, Barcelona, 1973 .

2. Kettelkamp, D.B.; Chao, M.S.: A Method for Quantitative Analysis of Medial and Lateral Compression Forces at the Knee During Standing. Clin Orthop; 83 March-April 1972; 202-213.

3. Maquet, P.G.: Biomechanics of the Knee; Springer Verlag; Berlin-Feidelberg: 59-67; 1976

4. Pavlov, H.; INSALL, J.M.: Surgery of the Knee. Churchill Livingstone Inc. New York, 1984.

5. Pichel, C.M.; Montoya, M.A.: Pathogenesis of Osteoarthritis of the knee. New Biomechanical Hypothesis.; Rev. Rhum.; num. special juin; 0744; 1981. 